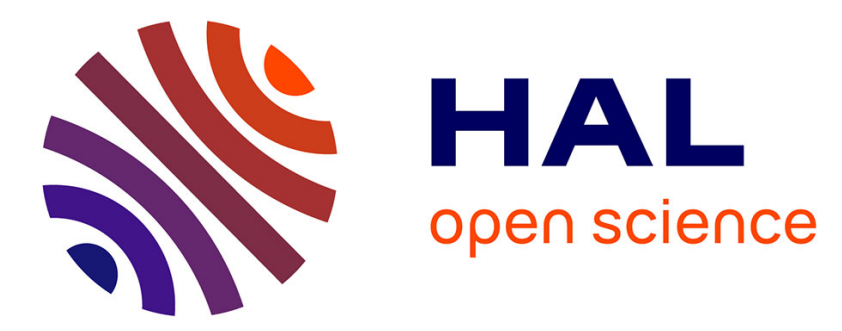

\title{
Un modèle pour comprendre l'influence de l'état de la recherche, des processus post-déposition et de l'attractivité sur la découverte des sites archéologiques dans le Kochersberg (Bas-Rhin, Alsace, Grand-est, France)
}

Charlène Morel

\section{To cite this version:}

Charlène Morel. Un modèle pour comprendre l'influence de l'état de la recherche, des processus postdéposition et de l'attractivité sur la découverte des sites archéologiques dans le Kochersberg (Bas-Rhin, Alsace, Grand-est, France). Archimède : archéologie et histoire ancienne, 2020, 7, pp.231-240. halshs02893739

\section{HAL Id: halshs-02893739 \\ https://shs.hal.science/halshs-02893739}

Submitted on 8 Jul 2020

HAL is a multi-disciplinary open access archive for the deposit and dissemination of scientific research documents, whether they are published or not. The documents may come from teaching and research institutions in France or abroad, or from public or private research centers.
L'archive ouverte pluridisciplinaire HAL, est destinée au dépôt et à la diffusion de documents scientifiques de niveau recherche, publiés ou non, émanant des établissements d'enseignement et de recherche français ou étrangers, des laboratoires publics ou privés. 


\section{ARCHIMÈDE N N 7}

\section{DOSSIER THÉMATIQUE : GESTES RITUELS. DE LA TRACE À L'INTERPRÉTATION}

\section{ACTUALITÉ DE LA RECHERCHE GÉOSCIENCES ET ARCHÉOLOGIE : INTERACTIONS, COMPLÉMENTARITÉS ET PERSPECTIVES}

114 Bruno GAVAZZI

Heureuses rencontres. Vers le développement d'approches intégrées en géosciences et archéologie

119 Jean-Paul BRAVARD

Dialogue interdisciplinaire : de I'unité stratigraphique aux interactions culture-environnement

129 Ferréol SALOMON

Les origines d'Ostie : quelles interactions avec la dynamique d'embouchure? (Delta du Tibre, Italie)

141 Quentin BORDERIE, Rowena Y. BANERJEA, Stéphane BONNET, Yannick DEVOS, Cristiano NICOSIA, Christophe PETIT, Ferréol SALOMON, Nathalie SCHNEIDER, Barbora WOUTERS, \& Patrice WUSCHER Géoarchéologies des contextes urbains : mieux comprendre les modalités de l'artificialisation des géosystèmes

158 Patrice WUSCHER, Christophe JORDA, Quentin BORDERIE, Nathalie SCHNEIDER \& Laurent BRUXELLES De la formation géologique à la tranchée : trouver et comprendre les sites archéologiques menacés par les travaux d'aménagement du territoire

176 Morgan MILLET \& Michel GUÉLAT

Les vestiges antiques de Rennaz-Noville (Vaud, Suisse) et leur contexte sédimentaire : nouvelles évidences de l'écroulement du Tauredunum

188 Théophile PIAU, François BÉTARD, Fabienne DUGAST, Gilles ARNAUD-FASSETTA \& Vincent VIEL Dynamique géomorphologique holocène et occupation humaine dans le bassin-versant de l'Eure (Bassin de Paris, France) : potentiels d'une approche géoarchéologique multiscalaire et diachronique

205 Dominique SCHWARTZ, Vincent ROBIN, Pierre ADAM, Philippe SCHAEFFER, Anne GEBHARDT, Pierre-Alexis HERRAULT, Benjamin KELLER, Daniele DAPIAGGI, Claire STEVENEL, Maxime THISS, Martine TRAUTMANN \& Damien ERTLEN

Les géosciences au service de l'archéologie agraire. Une étude de cas sur les rideaux de culture de Goldbach (68)

217 Étienne MANTEL, Stéphane DUBOIS, Jonas PARÉTIAS, Victor VISQUESNEL-SCHLOSSER, Corentin VOISIN, Bruno GAVAZZI \& Matthieu RICHARD

Étudier I'occupation d'une ville : les enjeux du PCR « Topographie générale et insertion territoriale de l'agglomération antique de Briga »

231 Charlène MOREL

Un modèle pour comprendre l'influence de l'état de la recherche, des processus post-déposition et de l'attractivité sur la découverte des sites archéologiques dans le Kochersberg (Bas-Rhin, Alsace, Grand-Est, France)

241 Lizzie SCHOLTUS

Spatialisation des découvertes, modélisation du passé. L'informatique au service de l'archéologie

254 Guillaume HULIN \& François-Xavier SIMON

Inrap et géophysique : vers une approche raisonnée

260 Hugo REILLER, Matthieu FUCHS, \& Bruno GAVAZZI

Approche multi-méthodes expérimentale pour l'étude d'un site d'occupation romaine et médiévale à Horbourg-Wihr

272 François-Xavier SIMON, Julien GUILLEMOTEAU, Guillaume HULIN, Joachim RIMPOT, Julien THIESSON \& Alain TABBAGH

De nouvelles perspectives pour les applications des méthodes électromagnétiques basse fréquence en archéologie

283 Rémy WASSONG \& Bruno GAVAZZI

Apport des prospections magnétiques haute résolution à la compréhension d'un habitat protohistorique : l'exemple du site de hauteur fortifié du Maimont 


\title{
UN MODĖLE POUR COMPRENDRE L'INFLUENCE DE L'ÉTAT DE LA RECHERCHE, DES PROCESSUS POST-DÉPOSITION ET DE L'ATTRACTIVITÉ SUR LA DÉCOUVERTE DES SITES ARCHÉOLOGIQUES DANS LE KOCHERSBERG (BAS-RHIN, ALSACE, GRAND-EST, FRANCE)
}

\author{
Charlène MOREL ${ }^{1, *}$ \\ ${ }^{1}$ UMR 7044 Archimède - Université de Strasbourg \\ *charlene.e.morel@gmail.com
}

RÉSUMÉ

Dans le cadre d'une étude portant sur les stratégies d'implantation des communautés agricoles, un modèle spatial est développé afin de mieux intégrer les biais de la carte de répartition des sites connus. Les paramètres inclus dans la modélisation sont l'état de la recherche archéologique, les processus post-déposition et un critère d'attractivité. L'objectif de ce modèle est de mieux comprendre quels facteurs favorisent l'implantation, la conservation, la découverte des vestiges et in fine la carte de répartition des sites archéologiques. Malgré quelques limites inhérentes à I'exercice de la modélisation spatiale, le

MotS-CLÉs

Modélisation,

SIG,

archéologie spatiale,

Kochersberg,

géoarchéologie,

taphonomie. modèle produit de nouveaux résultats qui sont détaillés via un exemple concernant la micro-région du Kochersberg en Alsace (Grand-Est, France).
As part of a study focusing on the settlement strategies of farming communities, a spatial model is developed in order to overcome the repartition map's biases. This model integrates as parameters the state of archaeological investigation, the taphonomical processes and an attractiveness criterion. The aim of the model is to understand which parameters influence the establishement of settlements as well as the conservation and discovery of their remains. Although this model has a few limitations, it produces new results that are detailed through the case study of the Kochersberg region in Alsace (Grand-Est, France).
KeYWORDS Modeling, GIS spatial archaeology, Kochersberg, geoarchaeology, taphonomy. 
La répartition des vestiges archéologiques est au cœur de toutes les problématiques en archéologie spatiale. Les méthodes utilisées afin de répondre à ces questionnements depuis les années 1950 reposent sur la cartographie des sites avec une prise en compte très légère voire inexistante des biais inhérents à l'archéologie tels que l'état de la recherche archéologique et les processus post-déposition [1]. Depuis 2007, les cartes de confiance archéologiques sont couramment utilisées [2], mais elles ne suffisent pas à prendre en considération tous les biais archéologiques. En effet, même si les biais liés à l'état de la recherche sont pris en compte, ceux liés aux processus post-déposition tels que l'érosion ou le recouvrement sédimentaire ne le sont pas. Afin de combler ce manque, un modèle spatial est établi en prenant en compte à la fois les processus taphonomiques, l'état de la recherche archéologique ainsi que l'attractivité potentielle estimée. Cet article traite à la fois de l'élaboration de cette modélisation et de son application à la micro-région lœssique du Kochersberg, au nord-ouest de Strasbourg (BasRhin, Alsace, Grand-Est, France).

\section{MÉTHODOLOGIE}

Ce travail vise à étudier l'occupation et l'exploitation d'un milieu lœssique du Néolithique (environ 5600 avant notre ère) jusqu'au XII ${ }^{\mathrm{e}}$ siècle de l'ère commune. Les milieux lœssiques comptent parmi les plus fertiles du monde [3] et sont de ce fait peuplés tôt et intensément [4]. Les problématiques touchant à la répartition des sites archéologiques et plus particulièrement aux stratégies d'implantation sont sensibles à des biais tels que l'état de la recherche archéologique ou les effets de masques ou de destruction des processus post-déposition. Des outils palliant ces biais ont été créés à l'instar de la carte de confiance archéologique dont le but est d'estimer la fiabilité des corpus en fonction de l'état de la recherche archéologique et de la représentativité des données [5]. Il est cependant nécessaire de disposer d'un outil offrant plus de paramètres explicatifs quant à la répartition des sites que la carte de confiance seule qui ne prend pas en compte les processus post-déposition ainsi que les paramètres ayant une influence sur les stratégies d'implantation. Pour tenter de dépasser ces limites, il est proposé de développer un modèle spatial incluant l'état de la recherche archéologique, les processus post-déposition (érosion et recouvrement) et un paramètre plus social et directement lié aux populations étudiées : I'attractivité potentielle des secteurs [6]. En premier lieu, cette modélisation se fonde sur I'hypothèse que l'état de la recherche, les processus taphonomiques et l'attractivité influencent tous les trois la découverte des vestiges archéologiques. C'est pourquoi l'état de la recherche archéologique et les processus post-déposition sont pris en compte dans la modélisation. Cependant, ce n'est pas sur ces critères que les principaux tests sont effectués, mais sur celui de l'attractivité estimée, puisque le but est de mieux comprendre quelles stratégies d'implantation étaient mises en œuvre par les communautés agricoles du passé. L'hypothèse principale testée dans cet article est que ces communautés s'installaient de façon préférentielle sur les sols les plus fertiles. Ainsi, cette démarche vise à étudier en particulier l'impact de la fertilité des sols sur l'implantation des populations. Pour évaluer la fiabilité du modèle, les résultats sont comparés au corpus archéologique afin de vérifier d'une part que les trois critères considérés influencent bien tous la découverte des vestiges et ensuite de tester l'impact en particulier de la fertilité sur le peuplement. La zone d'étude se situe en Alsace, dans le Bas-Rhin, au nord-ouest de Strasbourg ; il s'agit du placage lœssique du Kochersberg (fig. 1).
[1] DJINDJIAN 2011 (notamment le chapitre 17, p. 485-503).

[2] Notamment grâce aux travaux de l'équipe d'ArchaeDyn : Ostir et al. 2008.

[3] CATT 2001.

[4] BAKELS 2009.
[5] Ostir et al. 2008.

[6] Ce critère se retrouve sous plusieurs formes dans la bibliographie traitant des périodes spécifiques, comme par exemple JeUnesse 1980, PLouin \& WOLF 1989, CAROzZA et al. 2017 et THOMANN 2008 sans cependant n'avoir jamais été testé. 


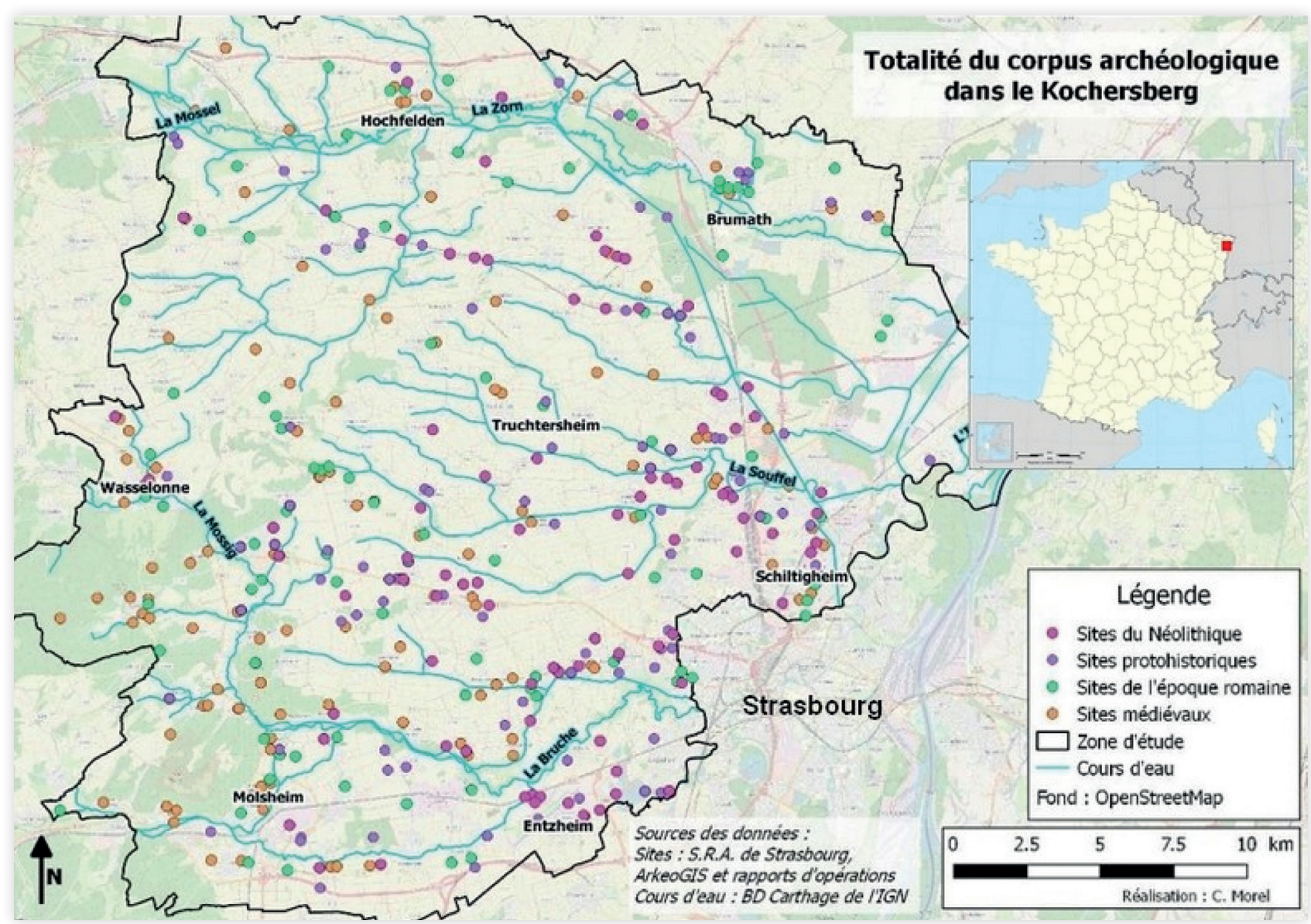

Figure 1 : zone d'étude au sein du Kochersberg et corpus archéologique (DAO : C. Morel).

En détail, cette modélisation prend en compte les données suivantes :

- La totalité de la documentation archéologique de la micro-région étudiée afin d'établir un état de la recherche et de travailler sur les questions de confiance et de fiabilité de la donnée ;

un modèle numérique de terrain (MNT - réalisé à partir des données de la BD Topo de I'IGN au pas de $25 \mathrm{~m}$ [7]) qui sert à calculer une carte des formes du paysage afin de travailler sur l'érosion et le recouvrement sédimentaire ;

la carte pédologique (ici, celle au 1/100000è issue des travaux de I'Association pour la relance agronomique en Alsace - ARAA [8]), utilisée afin de déterminer la fertilité relative d'un sol par rapport à ses voisins à l'aide des caractéristiques physiques et chimiques.

Ces données géoréférencées sont ensuite introduites dans un SIG afin de pouvoir effectuer des opérations sur les données et d'en extraire des informations pertinentes pour le modèle à l'aide d'outils de traitements simples et clef en main. De fait, ces différentes données ne sont généralement pas produites pour l'archéologie et il convient de faire preuve de discernement dans leur utilisation ainsi que de comprendre leurs limites. Par exemple, la carte des sols de I'ARAA est produite pour et par des agronomes et comporte des informations ayant trait aux sols actuels.

La première étape de la modélisation consiste à fixer les paramètres de l'état de la recherche et des processus sédimentaires à travers des cartes de répartition de ces phénomènes. La carte de l'état de la recherche archéologique est créée au moyen de plusieurs couches vectorielles à partir de toute la documentation archéologique (rapports de prospection, de fouilles mais aussi les diagnostics négatifs) ; chacune représentant l'étendue d'une opération archéologique. Toutes les opérations ont été prises en compte sans considérer leur nature ou leur résultat [9]. Toutes ces couches sont ensuite

[7] Pour plus d'informations sur la BD Topo et les conditions d'accès : https://geoservices.ign.fr/ressources_documentaires/Espace_documentaire/BASES_ VECTORIELLES/BDTOPO/DC_BDTOPO_3-0.pdf

[8] http://www.araa-agronomie.org/

[9] Puisqu'il s'agit ici de cartographier l'état de la recherche, les diagnostics négatifs sont tout aussi utiles que ceux ayant livré des vestiges. 
regroupées dans le but d'obtenir une carte répondant à la question « Où les archéologues ont-ils cherché des vestiges ? ». On nomme cette couche la carte de fiabilité.

Ensuite, les processus sédimentaires sont cartographiés. Comment est-il possible de rendre compte de l'érosion et/ou du recouvrement sédimentaire dans une micro-région d'environ 700 kilomètres carrés ? La solution choisie est de procéder à une simplification de ces phénomènes. Pour se faire, on calcule un TPI (Topographic Position Index) à partir du MNT. Cet outil ainsi que le suivant proviennent de la boîte à outils « Topography Tools » pour Arcview dans sa version 1.3 [10]. Cette opération consiste à calculer la position topographique relative d'une cellule par rapport à ses voisines. Le voisinage utilisé est de neuf cellules, soit neuf pixels (ou 25 mètres par cellule) de chaque côté. À partir de ce TPI, il est alors possible de réaliser une classification en forme du paysage à l'aide d'un algorithme de «landform classification » [11]. Cet algorithme permet de classer chaque cellule du raster selon un code de 1 à 10 en fonction de la pente relative avec les cellules avoisinantes. Cela permet ainsi de mieux appréhender la topographie du secteur. Afin de simplifier le modèle, ces dix classes sont regroupées - via l'addition de certaines classes dont la topographie est proche - en trois qui correspondent aux positions topographiques suivantes : plaines, pentes ou sommets et fonds de versants ou de vallons. Ces classes correspondent alors à des classes de pentes relatives en fonction de la topographie extra-locale, c'està-dire seulement les neuf cellules voisines, et pas à l'échelle de tout le Kochersberg, Ce qui permet une meilleure cartographie de phénomènes aussi locaux que l'érosion. Il est alors possible d'attribuer - en fonction des classes de topographie - la valeur de « recouvrables, n'ayant probablement pas subi d'érosion ou de recouvrement sédimentaire majeur 》 aux zones planes, « érodées 》 aux zones en pente et aux sommets et « recouverts $\gg$ aux fonds de vallons. Cette attribution se fonde sur le fonctionnement général des processus

[10] JENNESS 2006.

[11] Weiss 2001, Jenness 2006, Mokarram 2015. [12] ERTLEN et al. 2012.

[13] https://docs.qgis.org/2.18/fr/docs/user_manual/ processing_algs/qgis/vector_overlay_tools.html

[14] Cela rend le modèle plus simple à mettre en œuvre mais n'est pas indispensable à l'utilisation de l'outil d'union. sédimentaires au sein du Kochersberg [12]. On obtient ainsi une carte simplifiée des phénomènes post-déposition.

La troisième carte réalisée est celle de l'attractivité potentielle des secteurs pour les populations étudiées, soit la fertilité dans le cadre de cet article. La fertilité relative est établie à l'aide de la carte des sols de I'ARAA évoquée précédemment. Les caractéristiques présentes pour chaque type de sol de la classification de I'ARAA ont été étudiées afin de déterminer lesquelles pouvaient déjà exister aux époques anciennes, telles que la texture, la réserve utile et les propriétés chimiques du matériau parental. Le but n'était pas tant de pouvoir quantifier la fertilité réelle de chaque sol que de pouvoir attester de la productivité relative de ceux-là, c'est-àdire leur fertilité par rapport aux sols voisins. Le résultat obtenu est le classement de chaque type de sol dans une classe de productivité : très fertile, moyennement fertile et peu à pas productif.

Les cartes sont ensuite fusionnées les unes aux autres au sein du SIG afin d'obtenir une carte représentant simultanément les trois critères ; l'outil « Union » du logiciel QGIS est utilisé dans ce but [13]. Afin de représenter plus facilement les données, il est choisi d'avoir recours à un système de codes chiffrés ajoutés au sein d'une nouvelle colonne dans la table attributaire du résultat de I'union [14].

Ainsi, les processus post-déposition sont notés de la façon suivante : 100 pour «érodé », 200 pour « recouvert » et 300 pour «moindre impact »; l'état de la recherche quant à lui est noté ainsi : 010 pour « peu à pas fiable », 020 «moyennement fiable » et 030 « fiable»; enfin la fertilité relative de cette façon : 001 pour «peu à pas fertile », 002 pour «moyennement fertile » et 003 pour « très fertile ». Cette codification permet de regrouper les trois paramètres du modèle sous un code unique (fig. 2).

Par exemple, le code 200 / 010 / 003 - combiné en 213 - signifie que le secteur a subi un recouvrement sédimentaire, n'a pas été exploré par les archéologues et était probablement très attractif pour les communautés agricoles entre le Néolithique et le $\mathrm{XII}^{\mathrm{e}}$ siècle de l'ère commune. Au total, ce sont donc 27 combinaisons qu'il est possible d'obtenir au sein du modèle, de 111 pour une zone érodée, sans recherche archéologique et pas attractive à 333 pour un secteur n'ayant probablement pas subi d'érosion ou de recouvrement sédimentaire, bien connu archéologiquement et potentiellement très attractif. Ces combinaisons sont trop nombreuses pour être cartographiées de façon lisible. C'est pourquoi elles ont été contractées en « additionnant » les trois 


\begin{tabular}{|c|c|c|c|c|}
\hline $\begin{array}{l}\text { Combinaison } \\
\text { des critères }\end{array}$ & $\begin{array}{l}\text { Processus post- } \\
\text { déposition }\end{array}$ & $\begin{array}{c}\text { Fiabilité de la } \\
\text { recherche } \\
\text { archéologique }\end{array}$ & Fertilité relative & $\begin{array}{l}\text { Contraction } \\
\text { des critères }\end{array}$ \\
\hline 111 & Erosion & Mauvaise & Peu à pas fertile & 3 \\
\hline 112 & Erosion & Mauvaise & Moyennement fertile & 4 \\
\hline 121 & Erosion & Moyenne & Peu à pas fertile & 4 \\
\hline 211 & Sédimentation & Mauvaise & Peu à pas fertile & 4 \\
\hline 113 & Erosion & Mauvaise & Très fertile & 5 \\
\hline 122 & Erosion & Moyenne & Moyennement fertile & 5 \\
\hline 131 & Erosion & Très bonne & Peu à pas fertile & 5 \\
\hline 212 & Sédimentation & Mauvaise & Moyennement fertile & 5 \\
\hline 221 & Sédimentation & Moyenne & Peu à pas fertile & 5 \\
\hline 311 & Moindres & Mauvaise & Peu à pas fertile & 5 \\
\hline 123 & Erosion & Moyenne & Très fertile & 6 \\
\hline 132 & Erosion & Très bonne & Moyennement fertile & 6 \\
\hline 213 & Sédimentation & Mauvaise & Très fertile & 6 \\
\hline 222 & Sédimentation & Moyenne & Moyennement fertile & 6 \\
\hline 231 & Sédimentation & Très bonne & Peu à pas fertile & 6 \\
\hline 312 & Moindres & Mauvaise & Moyennement fertile & 6 \\
\hline 321 & Moindres & Moyenne & Peu à pas fertile & 6 \\
\hline 133 & Erosion & Très bonne & Très fertile & 7 \\
\hline 223 & Sédimentation & Moyenne & Très fertile & 7 \\
\hline 232 & Sédimentation & Très bonne & Moyennement fertile & 7 \\
\hline 313 & Moindres & Mauvaise & Très fertile & 7 \\
\hline 322 & Moindres & Moyenne & Moyennement fertile & 7 \\
\hline 331 & Moindres & Très bonne & Peu à pas fertile & 7 \\
\hline 233 & Sédimentation & Très bonne & Très fertile & 8 \\
\hline 323 & Moindres & Moyenne & Très fertile & 8 \\
\hline 332 & Moindres & Très bonne & Moyennement fertile & 8 \\
\hline 333 & Moindres & Très bonne & Très fertile & 9 \\
\hline
\end{tabular}

Figure 2

Classes obtenues au sein du modèle spatial.

(doc : C.Morel). chiffres ensemble. La combinaison 111 devient alors 3, 123 devient 6, etc. Les combinaisons sont indiquées dans la dernière colonne du tableau présenté en figure 2. Cette opération permet de n'obtenir que sept classes plus cartographiables qui correspondent à des niveaux de probabilité de présence de sites dans le cas où I'hypothèse d'indépendance et de poids équivalent des paramètres du modèle est juste. Ainsi, la comparaison de la carte de la répartition connue [15] des sites archéologiques avec les cartes à sept classes et avec les histogrammes des vingt-sept classes permet d'explorer les différentes hypothèses.

\section{RÉSULTATS}

La figure suivante (fig. 3) montre la répartition des sites du Néolithique au XII ${ }^{\mathrm{e}}$ siècle de l'ère commune sur la carte de prédiction de la répartition des sites à sept classes simplifiées. On remarque sur cette carte que les zones aux scores les plus hauts (7 à 9) concentrent la majorité des sites tandis que très peu de sites ne sont présents dans les zones aux scores les plus bas (3 à 5), ce qui indique que les trois paramètres influencent bien la présence des sites. Néanmoins, il ne semble pas y avoir une répartition linéaire en fonction du score simplifié. Ce constat est confirmé par l'histogramme présentant la densité des sites au $\mathrm{km}^{2}$ au sein de chacune des vingt-quatre classes (fig. 4) [16].

On peut alors remarquer que dans la plupart des cas où deux des critères sont dans leur condition jugée la plus favorable (soit un 3) - classes 133, 233, 331, 332 -, la densité des sites est supérieure à la moyenne au sein du Kochersberg. La seule exception est la classe 323. On note aussi que certaines combinaisons sont plus favorables

[15] L'expression « répartition connue » est utilisée par opposition à la répartition réelle des sites qui, elle, est inconnue.

[16] Sur les 27 possibilités théoriques, seules 24 ont été trouvées sur le terrain avec la fertilité relative comme critère d'attractivité potentielle, il y a donc trois classes sans occurrence dans la zone. Il s'agit des classes 113,213 et 313. 


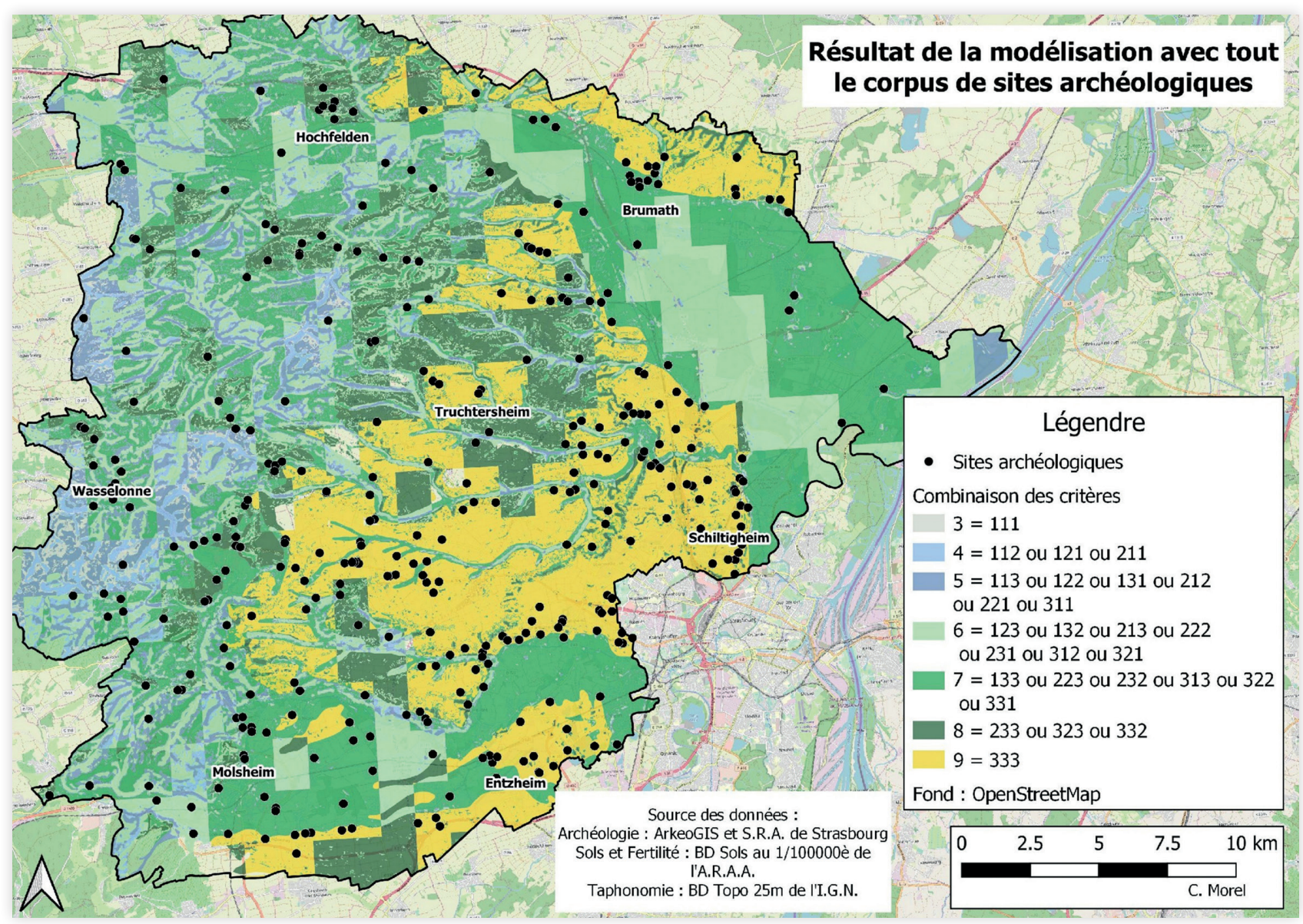

Figure 3

Carte des sites archéologiques sur les classes obtenues avec le modèle (DAO : C. Morel).

\section{Densité des sites au $\mathbf{~ k m}^{2}$ au sein des 24 classes du modèle}

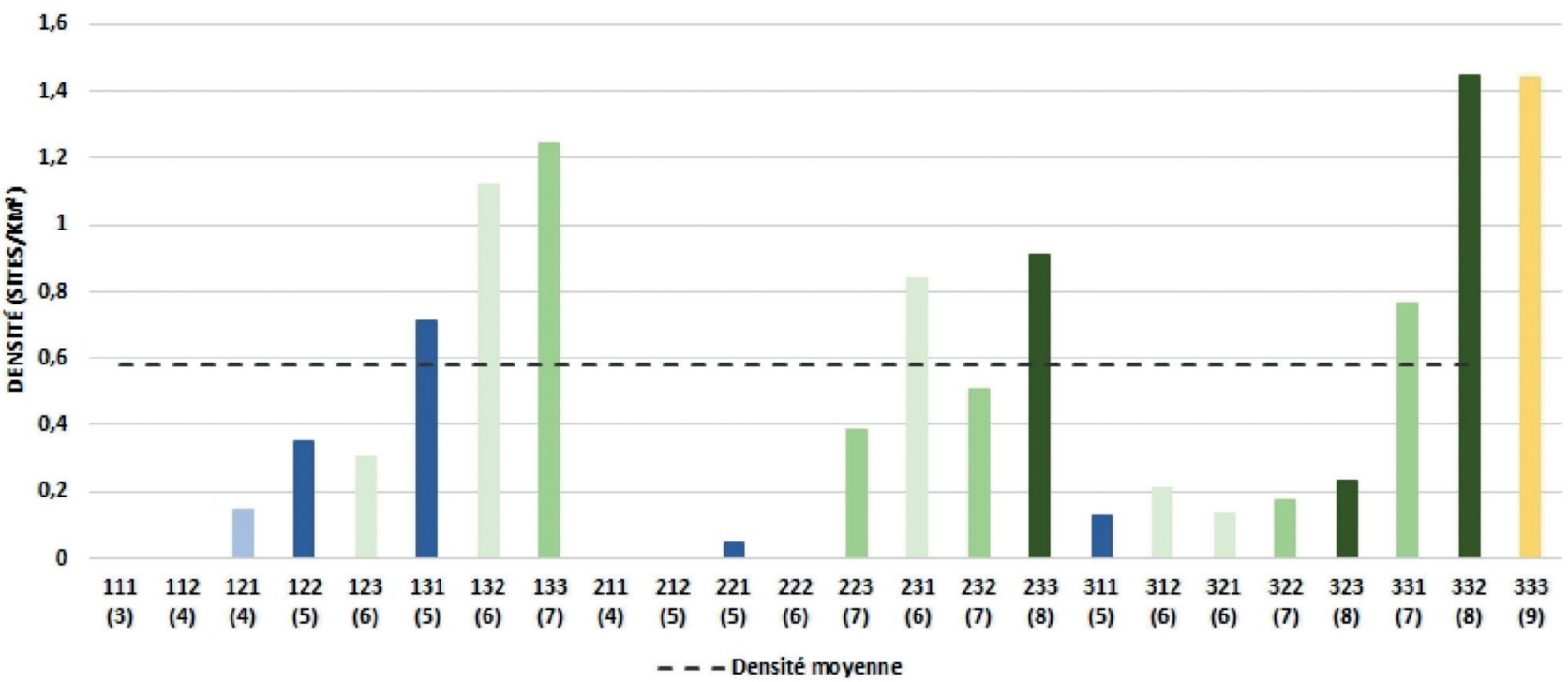

Figure 4

Histogramme présentant la densité des sites archéologiques au $\mathrm{km}^{2}$ au sein des vingt-quatre classes du modèle (doc : C. Morel). 
que d'autres malgré un score agrégé plus faible, par exemple la classe 132 (6) qui est plus peuplée que les classes 232 (7), 233 (8) ou encore 331 (7). On ne peut néanmoins pas remarquer de facteur significativement plus impactant que les autres. Ce résultat permet $\mathrm{d}^{\prime}$ affirmer que les trois critères pris en compte influencent bien les chances de découvrir des vestiges, mais qu'ils ne s'avèrent pas indépendants et de même poids relatif à l'échelle du corpus entier. Il apparaît en effet qu'un bon état de la recherche, peu de recouvrement et d'érosion et des sols très fertiles sont des critères nécessaires à la découverte de vestiges dans le Kochersberg, mais que d'autres paramètres impactent la distribution des sites de façon générale.

Une fois cette influence des trois paramètres validée, il est possible d'aller plus loin dans les pistes de réflexion ouvertes grâce à cette modélisation. On peut distinguer les sites de différentes périodes au sein du modèle afin de voir $s^{\prime}$ il existe des distinctions dans la façon dont les critères influencent la découverte des vestiges de chacun d'entre eux. Afin de pouvoir distinguer clairement si des différences existent entre les périodes, les plus extrêmes du corpus, c'est-à-dire les sites du Néolithique (c. 5600 - 2200 avant l'ère commune) et du Moyen Âge (du Ve siècle au XII siècle de l'ère commune) sont comparés. La carte suivante représente ces deux jeux de données sur le fond résultant de la modélisation (fig. 5).

Visuellement, il semble que les sites médiévaux (en rouge sur la carte) sont moins systématiquement dans les plages favorables que les sites du Néolithique. Ce phénomène est principalement visible dans le sud-ouest du Kochersberg.

La comparaison des histogrammes de répartition (fig. 6) confirme cette tendance : les sites médiévaux occupent un grand nombre de classes et sont présents dans pratiquement toutes les configurations à l'exception quasi-systématique de celles dont le premier chiffre est un 2, soit les classes ayant subi un recouvrement sédimentaire. Dans ces cas-là, il est nécessaire que la recherche archéologique soit très fiable (le second chiffre est un 3) pour que des sites du Moyen Âge soient découverts. Ce constat vis-à-vis du recouvrement sédimentaire est le même pour le Néolithique. Par contre, les sites du Néolithique sont globalement plus présents quand un maximum de critères sont réunis

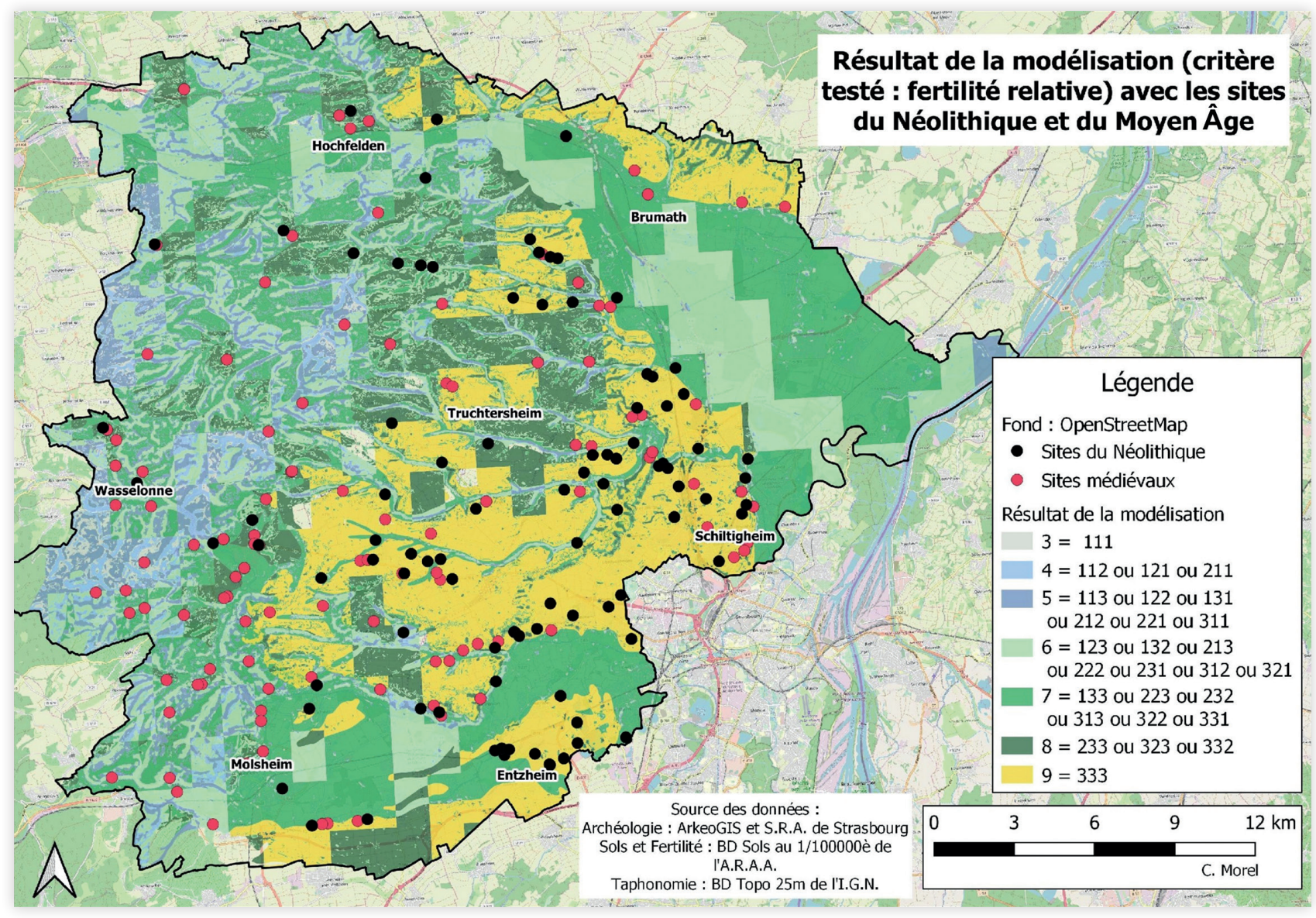

Figure 5

Sites du Néolithique et du Moyen Âge sur les classes du modèle (DAO : C. Morel). 


\section{Densité des sites du Néolithique et du Moyen Âge sur chaque classe de la modélisation}

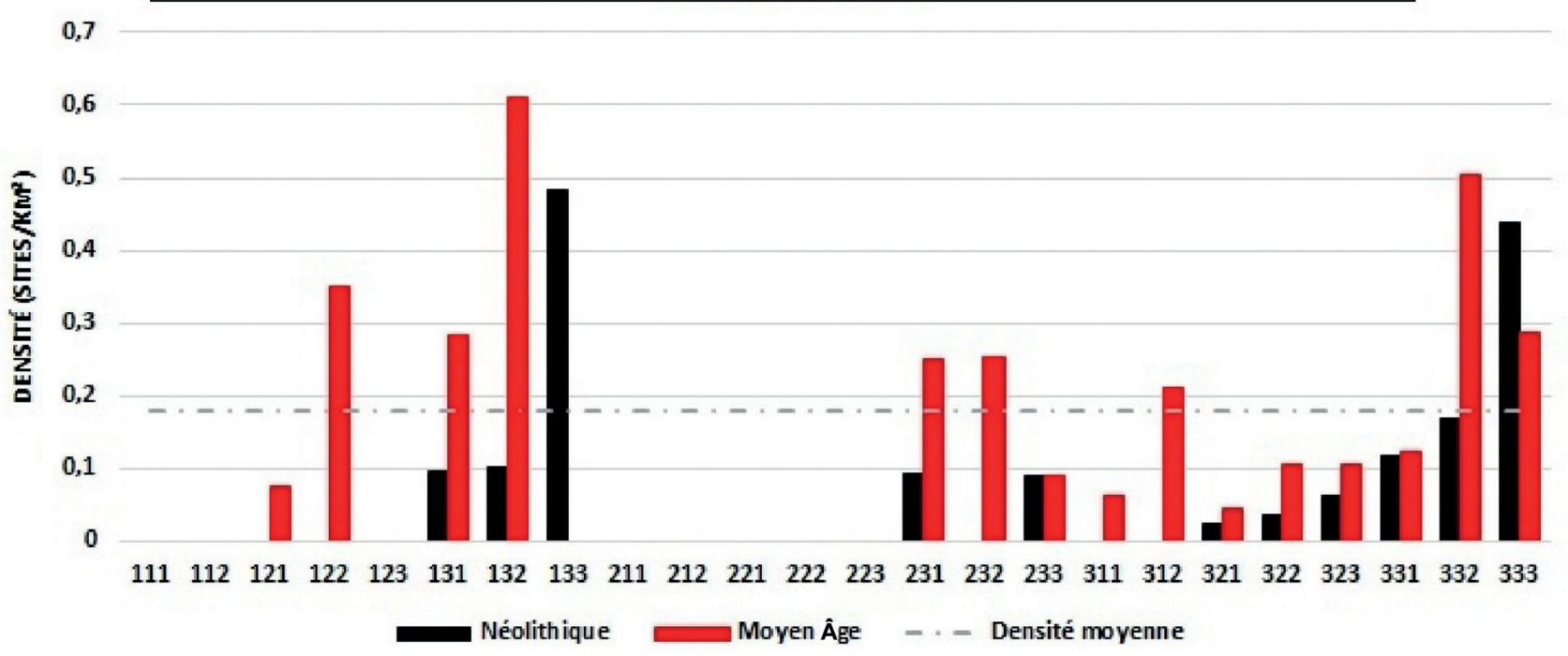

Figure 6

Histogramme des densités de sites néolithiques et médiévaux au $\mathrm{km}^{2}$ sur les vingt-quatre classes du modèle (doc : C. Morel).

et assez peu présents dans les deux premiers tiers du diagramme, à l'exception d'un pic au sein de la classe 133, qui signifie de l'érosion, une excellente recherche et les sols les plus fertiles (fig. 6). On note également que pour les sites néolithiques, lorsque le couple des deux premiers paramètres est assez bon $(32,33)$, la répartition semble fonction du troisième paramètre (la fertilité).

Il est alors possible d'en déduire que la découverte de vestiges médiévaux est moins dépendante des critères utilisés que celle des sites néolithiques. À quoi cette différence est-elle due ? La découverte de sites médiévaux dépend-elle de la même manière de chaque critère ? Et, plus concrètement, le modèle qui fonctionne bien avec les sites du Néolithique (et avec la totalité du corpus de sites du Kochersberg), est-il mal adapté en ce qui concerne les sites médiévaux ? Cela pousse aussi à s'interroger sur la façon dont le modèle a été construit : aurait-il fallu considérer que l'érosion a moins d'impact que le recouvrement sédimentaire sur les chances de découvrir des sites archéologiques dans le Kochersberg, et donc de noter le recouvrement en 1 et l'érosion en 2 ? Les résultats semblent l'indiquer.

En ce qui concerne l'attractivité des sols les plus fertiles, le modèle montre qu'au Néolithique, ce sont eux qui portent la vaste majorité de l'occupation. Ce résultat vient renforcer l'hypothèse d'une dépendance de l'implantation à la fertilité des sols. Cependant, ce n'est plus le cas au Moyen Âge où la carte tout comme I'histogramme montre un affranchissement des sites vis-à-vis des sols les plus fertiles. Ce résultat nouveau permet de mettre en avant une mutation - qui a eu lieu entre la fin du Néolithique et environ 450 de l'ère commune - dans les stratégies d'implantation des communautés d'agriculteurs. Plusieurs hypothèses peuvent être avancées pour expliquer cela : un changement dans les modes agricoles (plus extensifs, avec des habitats aux marges des sols très fertiles ainsi que ces derniers soient totalement libres pour l'agriculture), ou un changement plus large des modes de vie, avec I'utilisation plus large de produits importés par exemple.

\section{DISCUSSION}

La modélisation spatiale des trois critères qui, selon I'hypothèse de départ, favorisent le plus la découverte des sites archéologiques, offre un nouvel outil. Ce dernier permet d'attester des conditions les plus favorables - au sein d'un secteur donné à la découverte des sites archéologiques de différentes périodes. Cela offre un fort potentiel pour la création de modèles prédictifs et en termes de prescriptions archéologiques dans la région, bien qu'il ne s'agisse pas du sujet de cet article. Le modèle permet aussi de réfléchir à la répartition connue des sites, à ses biais, à sa logique, etc. Les simplifications réalisées pour la modélisation sont à la fois 
son point fort et ses limites. D'un côté, le modèle est totalement adaptable à n'importe quel secteur à I'aide de données relativement aisées à obtenir (un MNT, les données sur la recherche archéologique et une carte des sols par exemple) et permet l'obtention de résultats rapides. Cet atout n'est pas négligeable puisqu'il permet de rendre plus courante la prise en compte des biais inhérents à l'archéologie. Cependant, ces simplifications peuvent limiter la validité des résultats de la modélisation. En effet, les critères utilisés tels que les processus post-déposition peuvent être très locaux et de ce fait pas toujours aisés à cartographier à l'échelle d'une micro-région comme le Kochersberg. Ces phénomènes extra-locaux peuvent être à l'origine d'anomalies dans les résultats de la modélisation. Cela n'enlève rien à la pertinence du modèle, mais doit être gardé à l'esprit afin de réaliser, le cas échéant, les vérifications locales qui s'imposent. Dans tous les cas, une bonne connaissance du secteur est recommandée avant la mise en place d'une telle modélisation. Les résultats que produit cet outil nécessitent dans tous les cas l'interprétation du chercheur et la vérification des anomalies, voire un ajustement des critères utilisés.

\section{CONCLUSION}

Un modèle spatial a été créé afin de mieux comprendre quels critères influencent le plus la capacité des archéologues à mettre au jour les vestiges archéologiques. Cette modélisation prend en compte les processus post-déposition, l'état de la recherche archéologique et l'attractivité potentielle estimée des secteurs. Le résultat de la modélisation prend la forme d'une carte qui montre que ces trois critères jouent un rôle majeur dans la « découvrabilité » des vestiges. Les tests montrent que la capacité des archéologues à découvrir des sites du Néolithique est plus dépendante des trois facteurs que pour les sites du Moyen Âge où I'influence des processus post-déposition mérite d'être nuancée et celle la fertilité relative niée. Cela montre les limites d'un modèle fondé sur la simplification de phénomènes potentiellement très locaux et la nécessité de vérifier sur le terrain tout résultat qui apparaîtrait comme une anomalie. Pouvoir mettre en avant les mutations des stratégies d'implantation grâce à la modélisation permet de construire de nouvelles hypothèses à leur sujet.

\section{REMERCIEMENTS}

Le modèle a été construit à l'aide de Damien Ertlen et de Pierre-Alexis Herrault, du Laboratoire Image, Ville et Environnement (UMR 7362) que je remercie ici.

\section{BIBLIOGRAPHIE}

ARAA s. d. « À propos de l'ARAA ». ARAA. Consulté le 16 mars 2020. http://www.araa-agronomie.org/.

BAKELS, Corrie C., 2009, The Western European Loess Belt, Leiden.

CARozza, Laurent (éd.), 2017. L'habitat et l'occupation des sols à l'âge du Bronze et au début du premier âge du Fer, Paris (Recherches archéologiques 12).

CATT, John A., 2001, « The agricultural importance of loess », Earth-Science Reviews 54/1, p. 213-229.

DuIndjIAN, François, 2011, Manuel d'archéologie, Paris (Collection U Archéologie).

Ertlen, Damien, Gebhardt, Anne, Schneider, Nathalie, Durand, Frédérique, Thomas, Yohann, Michler, Matthieu, SchneIKert, François, BoËs, Eric, \& SchwarTz, Dominique, 2012, « Antropisation et érosion agraire dans un paysage loessique (Bas-Rhin, France)», dans Frédérique Bertoncello \& Frank Breamer (dir.), Variabilités environnementales, mutations sociales : nature, intensités, échelles et temporalités des changements, XXII rencontres internationales d'archéologie et d'histoire d'Antibes, 20-22 octobre 2011, Antibes, p. 85-92.

Jenness, Jeff, 2006, Topographic position index (tpi_jen.avx) extension for ArcView 3.x, (version 1.3a), Jenness Enterprises. 
JeUnesse, Christian, 1980, «Le peuplement rubané de Basse-Alsace : aspects géographiques », dans Le Rubané d'Alsace et de Lorraine : état des recherches 1979, Association d'études préhistoriques et protohistoriques d'Alsace, Strasbourg, p. 85-101.

Mokarram, Marzieh, Gholamreza, Roshan \& Saeed, Negahban. 2015, « Landform Classification Using Topography Position Index (Case Study: Salt Dome of Korsia-Darab Plain, Iran) », Modeling Earth Systems and Environment, 1/4, https://doi.org/10.1007/s40808-015-0055-9.

Ostir, Kristof, Kokalu, Ziga, Saligny, Laure, Tolle, Florian, \& Nuninger, Laure, 2008, « Confidence maps : a tool to evaluate archaeological data's relevance in spatial analysis », dans Axel Posluschny, Karsten Lambert \& Irmela Herzog (éd.), Layers of Perception, Proceedings of the 35th International Conference on Computer Applications and Quantitative Methods in Archaeology, Berlin 2-6 April 2007, Berlin, p. 272-277.

QGIS, s. d., «Recouvrement de vecteur», Consulté le 7 avril 2020. https://docs.qgis.org/2.18/fr/docs/user_manual/ processing_algs/qgis/vector_overlay_tools.html.

ThomanN, Marcel, 2008, «Le Kochersberg au Haut Moyen Âge, recherches, thèses et hypothèses », Revue d'Alsace 138, p. 79-127.

WeIss, Andrew, 2001, «Topographic position and land-form analysis.pdf », Poster, Seattle.

Wolf, Jean-Jacques, 1989, «La Tène en Alsace : 450-50 av. J.-C. », dans Suzanne Plouin (éd.), L'Alsace celtique : 20 ans de recherches: Musée d'Unterlinden du 21 novembre 1989 au 18 février 1990, Musée historique de Haguenau du 3 mars au 28 mai 1990, Musée historique de Mulhouse du 15 juin au 28 octobre 1990, Colmar, p. 94-100. 